\title{
Multi-criteria Intelligent Algorithm for Supply Chain Management
}

\author{
Mahdi Jemmali ${ }^{1}$, Loai Kayed B.Melhim ${ }^{2}$, Mafawez Alharbi ${ }^{3}$ \\ Department of Computer Science and Information, \\ College of Science in Zulfi, Majmaah University, \\ Al-Majmaah 11952, Saudi Arabia
}

\begin{abstract}
In most societies, supply chain management and e-procurement processes, are one of the cornerstones of any economy and a primary influencer on people's lives. Providing these communities with their different commodity needs depends on a wide range of suppliers. Due to supplier's variety and diversity; the process of choosing the suitable supplier is considered the difficult and critical process, especially if this process is performed traditionally then decision making will be time and effort consuming to reach the desired results. To solve the problem of choosing the suitable supplier, this research suggests an intelligent algorithm, based on a given determinants that are specified by the decision maker or the customer. The proposed algorithm employs a set of intelligent formulas that will convert the predefined preferences into quantitative measurements. Quantitative measurements will be used to differentiate between different suppliers or between the set of given offers. The experimental results showed that $D 3 S$ model employed the given preferences and succeeded in selecting the most appropriate offer among the many presented offers by the available suppliers.
\end{abstract}

Keywords-Decision making; supplier; scoring; algorithms, supply chain management

\section{INTRODUCTION}

Supply chain performance is critical part of any supply chain process either in advanced or in emerging economies. The continuously developing technologies all over the globe can be part of the solutions that can enhance the supply chain performance. Governments have to employ these technologies by making some decisions in its public procurement system to increase its effectiveness; since supply chain performance is a key role in the development of socio-economics for any country [1]. The next main issue in supply chain management is the market itself. Recent markets are changeable and characterized as unpredictable and volatile. While customers and organizations requests may vary, changed or even canceled, there are a set of limitations that have to be considered such as reliable vs unreliable suppliers, product model varieties and the time-consuming supply processes [1], [2]. These parameters would make decisions for supplier evaluation and selection a challenging task.

For governments or supply chain organizations, choosing a suitable supplier is affected by a set of different factors, such as cost and reliability. Supplier evaluation and selection is not performed by reviewing a list of prices; the process of selection requires analyzing and considering many factors. Besides, the supplier selection process should consider the environmental and the socio-economic issues in order to perform the selection and evaluation process; to reach the best sustainable supplier, one that can enhance supply chain performance. Outsourcing initiatives create new challenges to the markets, the supply chain process in many countries is suppliers dependent; making it more critical when evaluating the performance of those suppliers. Supplier selection and evaluation requires the consideration of many factors that include multiple objectives and criteria [3].

\section{RELATED WORK}

Many research types have been performed in the field of supplier selection and evaluation, such as: adopting different approaches with different implementations and employing a lot of applied mathematics and methodologies [3]. Different researches resulted in many decision support tools that are based on multi-criteria, which is been presented as a decisions supporting tools [4], [5], [6] and [7].

Governments or organizations that are in charge of the supplier evaluation and selection process must present a set of specific requirements that should be met during the selection or the evaluation process. This problem has been addressed by [8], where the authors integrates fuzzy Analytic Hierarchy Process (AHP) for discriminant analysis and fuzzy goal programming to support decision making process. However, the separation of highly capable suppliers from less-capable ones are completely specified by the quality and the quantity of the data used to trained the proposed model. For the same field, [9] presented a fuzzy (AHP), a decision-making knowledge that can recognize or change the rules of order allocations. The presented approach was implemented to adapt with group of multi-criteria supplier selection problems, which groups order allocation with supplier selection for dynamic supply chains to deal with market variations. While in [10] the authors explored the literature review to initiate a decision support model that specifies the supplier main criteria (delivery, price, service and quality), then exploits experts' opinions to perform the ranking process and finally applies analytic hierarchy process $(A H P)$ for the supplier selection process.

In the process of decision making, decision makers must consider many factors and criteria. The diversity and differences of these factors and criteria, may complicate the decision-making process. Such difficulties is the balance between the cost and the quality of the required product or service. In such a case, multiple criteria decision-making $(M C D M)$ has to be used by the decision makers and many different criteria must be used to facilitate decision-making by the decision-maker, as well as, to be capable of measuring different aspects of the problem considered, besides 
the ability to distinguish between other available alternatives. The $M C D M$ method is very useful for decision makers to choose among alternative options when multiple criteria decision methods are applied. The usage of $M C D M$ can help to reduce the effort of users resulting from dealing with the huge amount of information that is integrated to create solutions to complex problems [11].Different approach was presented by [12] that combines threat perception and decision support systems. The authors presented a design overview of existing decision support system approaches to highlight their features/merits then to incorporates the missing parameters in the existing decision support systems to enhance these systems.

Using fuzzy and multiple criteria decision-making techniques was adopted by many researchers as shown in the reviews [13], [14]. For example [2] deployed two-stage fuzzy approach subjective measures to deal with order allocation and supplier selection problem. While [15] found that it is possible to choose a set of suited alternatives based on hybrid fuzzy decision making approach. Author in [16] presented decisionmaking problems based on fuzzy group and experts opinions, to present a solution that can adapt with conflicting quantitative and non-quantitative criteria of evaluation to determine the best alternative solution. In [17], authors presented organizational and technological perspective, to support the management of automated industry supply chain operations. The supplier selection process was performed based on the experts opinion to specify the important criteria by matching between subsidiary criteria and principal criteria to recognize the sub-criteria and relate them to the basic criteria.

Researches of the multi-criteria decision approaches was treated in [18]. In this review, the authors presented the studies that appeared in international journals in the years from 2000 to 2008. The reviewed paper focused on multi-criteria supplier and selection decision approaches. The presented approaches were analyzed to find the limitations by a critical study of the given approaches and then to determine which is the most widely used and adopted approaches in the supplier evaluation and selection problem. recently, authors in [7] presented an intelligent $D M P A$ model based on a set of multi-criteria preferences that can be adopted by decision makers, in supply chain management and e-procurement processes, to select the appropriate offer among the presented offers from many suppliers.

In different approach [19] implemented a framework for $E R P$ system selection with a set of thirty related features, and then evaluate the weight of each criterion by stepwise weight assessment ratio analysis ( $S W A R A)$. While the ranking of all the alternatives was performed by preference ranking organisation method for enrichment of evaluations (PROMETHEE).

To realize the impact of the barriers and benefits on the eprocurement adoption decision for e-procurement systems the authors in [20] used Interpretive Structural Modeling $(I S M)$ to describe any relations between the barriers and benefits of the barriers and benefits on the e-procurement selection process, then validate these relations by using Structural Equation Modeling $(S E M)$.

In a sophisticated decision support system, it is difficult to select and evaluate offers given by different suppliers. Because of the variant constraints and the contradictory variables that decision makers (governments or organizations) should consider before any decision is taken; an intelligent algorithm will be proposed to exploit a set of pre-selected determinants to evaluate and select the given offers to give decision makers the ability to choose the most offer that meet the selected determinants. The evaluation process will be performed based on multi-criteria determinants, given by the manager or by the customer, as it will be proven by the given experimental results.

The rest of this paper is structured as follows. The research problem definition is presented in Section 2, while the proposed decision support system model is explained by example in Section 3. In Section 4,the scoring of the $D 3 S$ algorithm will be presented. Section 5 presents experimental results and finally, conclusion will be presented in Section 6 .

\section{PROBLEM DEFINITION}

Decision making and supply chain management considered as important and critical tasks for any government and for any economic power. Given the importance of this topic, the presented research will highlight this problem by introducing a model that will deploy an intelligent algorithm based on a set of determinants selected in advance by the decision maker or the customer, the presented model will evaluate the supplier based on the given determinants by implementing these determinants into a group of variables. Each variable represents a certain criterion regarding the suppliers and the offers they present. The presented intelligent algorithm, the Decision Support and Selection System $(D 3 S)$ adapt with all the preselected determinants (cost, after sale service, compatibility, etc.) to derive the best supplier with the best offer among many presented suppliers and offers.

The manager is the only responsible who decide the limit of his requirements of each constraint. For this reason, we fix a threshold variable evaluated by the manager to measure the distance between offer and demand for each variable. The preselected determinants will be represented by a set of variables represented by vector $X$ where $X=\left\langle X_{1}, . ., X_{15}\right\rangle$. The vector $X$ is described by the following 15 elements:

- $X_{1}$ : Item name

- $X_{2}$ : Item maker

- $X_{3}$ : Item model

- $X_{4}$ : Required quantity

- $X_{5}:$ Number of trainees to use the item

- $X_{6}$ : Time required for training (Hours)

- $X_{7}$ : Max Budget (Dollar)

- $X_{8}$ : The time required to deliver the product (Days)

- $X_{9}$ : Warranty (Years)

- $X_{10}$ : After sale services (Years)

- $X_{11}$ : Available maintenance time (Days)

- $X_{12}$ : Available maintenance time (Hours)

- $X_{13}$ : Certificate of Origin (yes, no, $N A$ ).

- $X_{14}$ : Size of company $(M, L, S, N A)$

- $X_{15}$ : Company experience in the field of supply (Years).

Where,

Variables $X_{1}, \ldots, X_{6}$ implement products determinants specified by the customer and the variable $X_{7}$ represents 
budget limit. The variables $X_{8}, \ldots, X_{12}$ represent the services provided by the company. The variables $X_{13}, \ldots, X_{15}$ hold company details.

For the variable $X_{14}, S, M, L$ refers to small, medium company and large companies respectively. While for the variables $X_{13}$ and $X_{14}$ the value $N A$ represents "not applicable". For the variables $X_{13}$ and $X_{14}$, if the user chooses $N A$ then the corresponding variable will not have any preferred value i.e. will not be considered when applying the evaluation process.

Example 1: For a given company, the IT section needs to buy 20 printers with some preferences which are fixed as follows: Item name is printer, Item maker is HP, Item model is "Laser Jet Pro 400", Required quantity is 20, Number of trainees to use the item 3, Time required for training is 6 hours, Max Budget is $7000 \$$, The time required to deliver the product is 21 days, Warranty is 3 years, After sale services is 2 years, Available maintenance time 5 days and 8 hours, the Item must have Certificate of Origin, Size of company presenting the offer is not fixed as preference and the Company experience in the field of supply must be at least 4 years.

Based on the preferences given above for the customer, the variable $X$ is modeled as follows: $X_{1}=$ Printer, $X_{2}=H P$, $X_{3}=$ Laser Jet Pro 400, $X_{4}=50, X_{5}=3, X_{6}=6, X_{7}=$ $7000, X_{8}=21, X_{9}=3, X_{10}=2, X_{11}=5, X_{12}=8$, $X_{13}=y e s, X_{14}=N A$ and $X_{15}=4$.

The variables described in the vector $X$ are classified into four categories. Each category will be denoted by a vector as follows:

- Item: $X^{1}=\left\langle X_{1}, \ldots, X_{6}\right\rangle$

- $\quad$ Budget: $X^{2}=\left\langle X_{7}\right\rangle$

- Services: $X^{3}=\left\langle X_{8}, \ldots, X_{12}\right\rangle$

- Company: $X^{4}=\left\langle X_{13}, \ldots, X_{15}\right\rangle$

Thus, $X=\left\langle X^{1}, X^{2}, X^{3}, X^{4}\right\rangle$.

We denoted by:

- $j$ is the index offer

- $Y(j)$ the offer with $j$ index.

- $n$ the total number of presented offers.

- $k$ the index representing the given determinants.

The presented offer $j$ (before evaluation) will be denoted by $Y(j)=\left\langle Y_{k}(j)\right.$, with $\left.k \in\{1, \ldots, 15\}\right\rangle$. The vector $Y(j)$ can be written also based on the previous classification as $Y(j)=$ $\left\langle Y^{1}(j), Y^{2}(j), Y^{3}(j), Y^{4}(j)\right\rangle$.

\section{Decision Support System Model}

This section presents the mathematical model of the (D3S) by focusing on the decision variables which will form the basis of the evaluation process which will determine whether to continue evaluating or to reject the given offer.

\section{A. Decisive Variables}

The system must choose intelligently (considering the decisive variables) the best offer based on the given preferences specified by the customer. In this research only
$X_{1}, X_{2}$ and $X_{3}$ will be decisive variables, which means the first evaluation of the given offer $j$ will be executed based on these variables $\left(X_{1}, X_{2}\right.$ and $\left.X_{3}\right)$. Indeed, the first evaluation test will be: $\left(X_{1}=Y_{1}(j)\right.$ and $X_{2}=Y_{2}(j)$ and $\left.X_{3}=Y_{3}(j)\right)$ is true. Else, reject this offer with no more evaluations on the remaining variables.

Fig. 1 below shows the preliminary evaluation process based on decisive variables.

\section{B. Decision Process}

The preliminary process of $D 3 S$ is given in Fig. 1. Several components is presented to show the steps of the proposed algorithm to determine the best offer among an amount of offers obtained after research.

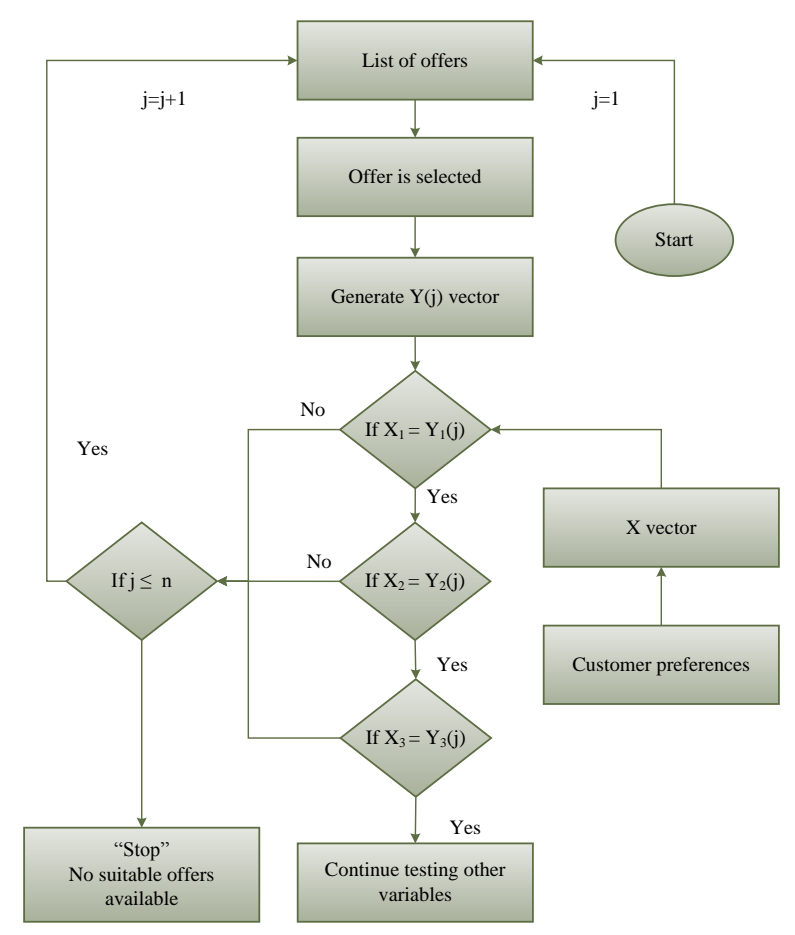

Fig. 1. The $D 3 S$ preliminary model

Fig. 1 consists of the following components:

- $\quad$ List of offers: the offers have been inserted and stored in the database and can be used to create the offers list.

- Offer is selected: after having the offers list in "list of offers" component, the system select the offer number.

- Generate $Y(j)$ vector: all vector element values of $Y_{i}(j)$ will be retrieved from the list of offers.

- Customer preference: this component present the preselected determinants.

- $X$ vector: the system generate a list contain all values of $X_{i}$ corresponding elements. This list will be presented in the vector $X$.

- If $X_{1}=Y_{1}(j)$ : This component examine the equality between $X_{1}$ and $Y_{1}(j)$. 
- If $X_{2}=Y_{2}(j)$ : This component examine the equality between $X_{2}$ and $Y_{2}(j)$.

- If $X_{3}=Y_{3}(j)$ : This component examine the equality between $X_{3}$ and $Y_{3}(j)$.

- If $i<n$ : This component test if the number of the total offers have been reached. If $i<n$ the system must increment the value of $i$ and restarted from the component "list of offers" with the new value of $i$.

- Continue test: If the decisive variables were tested and returned true, the system continue testing the remaining variables from $X_{4}$ to $X_{15}$

- Stop: if the total number of all offers have been reached, that mean there is no offer to test and the system must exit and returned the message "no offers are suitable based on the available determinants".

\section{SCORIng And Algorithms of the $D 3 S$}

This section explains how to define and derive the distance between a given offer and the specified threshold that will be determined based on the given determinants. the distance will

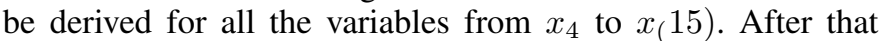
the final calculated score of an offer will be explained. Finally, the $D 3 S$ algorithms will be presented.

\section{A. Distance Formulation}

After finalizing decisive variables, we can separate these variables from the rest of the remaining variables. Thus, we will have a new sub-problem with 12 variables: $\left\{Y_{4}(j), \ldots, Y_{15}(j)\right\}$. The question is How to create a model that deploys $X_{4}$ and $X_{15}$ variables and can be used to derive the value of any given offer?

The first step is to specify any existing correlations between these 12 variables. This is can be performed by the application of t-students tests from SPSS software. The resulted values of t-students tests will be saved into $w_{i}$ variables, which will be the weight $w_{i}$ corresponding to each associated variable $X_{i}$ with $i \in I=\{4, \ldots, 15\}$. To measure the customer satisfaction rate a threshold for each $I$ variables must be determined this threshold represents a bound measuring the customer satisfaction for each variables. The defined threshold will be denoted by $T h_{i}$ the threshold of variable $X_{i}$ with $i \in I$.

Now after defining the threshold for all the variables, it is primordial to give the distance between the threshold and the given values of each offer $j$ variables with $i \in I$. The corresponding distance value will be in the interval of $[0-10]$. This distance will be denoted by $d_{i}^{j}$.

Proposition 1:

$$
d_{i}^{j}=\frac{Y_{i}(j)}{T h_{i}} \times 10
$$

For special case when $Y_{i}(j)>T h_{i}$, when the distance exceeds 10 . In this case we force $d_{i}^{j}$ to be 10 . This to guarantee that the best value is 10. Therefore, using Equation (1), the calculation of $d_{i}^{j}$ will be as follows:

$$
d_{i}^{j}= \begin{cases}\frac{Y_{i}(j)}{T h_{i}} \times 10 & \text { if } Y_{i}(j) \leq T h_{i} \\ 10 & \text { otherwise }\end{cases}
$$

Example 2: Assume that $j=20$ in this example. For the variable $X_{4}$, the user requires $t h_{4}=50$, however the company offer is $Y_{4}(20)=30$. In this case the distance based on Equation 2 will be

$d_{4}^{20}=\frac{Y_{4}(20)}{T h_{4}} \times 10=\frac{30}{50} \times 10=6$, so the evaluation of the offer for the variable $X_{4}$ is 6 out of 10 .

All distances $d_{i}^{j}$ will be calculated by the $D 3 S$ model. For the variable $x_{7}$ distance $d_{7}^{j}$ and the variable $x_{14}$ distance $d_{14}^{j}$, there are some extra calculations and will be explained in the subsections below.

1) Budget distance: For the variable $Y_{7}(j)$, we proceed with inverse evaluation. This means that when the budget will be more higher, the evaluation will be more lower and vice versa. For the budget calculations, specify the special evaluation based on the sign of $T_{B}^{j}$ in the following equation of budget test, and defined as:

$$
T_{B}^{j}=1-\frac{Y_{7}(j)}{T h_{7}} .
$$

To specify weather the offer is expensive or meet the specifications determined by the customer, this will be derived from $T_{B}^{j}$ as explained in Remark 4.

\section{Remark 1:}

$$
T_{B}^{j}\left\{\begin{array}{l}
>0 \text { is good than preferences } \\
=0 \text { exactly equal to preferences } \\
<\text { expensive than preferences }
\end{array}\right.
$$

Example 3: For offer 20, assume that the maximum budget $Y_{7}(20)=40,000$ and the threshold $T h_{7}$ determined by the head manger is 30,000. In this case the presented offer exceeds the available budget by 10,000. Applying Equation 3 we have: $T_{B}^{20}=1-\frac{Y_{7}(20)}{T h_{7}}=1-\frac{40,000}{30,000}=1-1.333=-0.333$. Since $T_{B}^{20}=-0.333<0$ and referring to Equation 4 the system can deduce that the offer does not meet the given preferences.

Besides the weight factor in the model, we can add an other parameter related essentially to the budget because of the importance of this variable. To provide the customer with more options regarding the budget, so that to increase the effectiveness of the budget variable. we propose to introduce the multiplicity factor denoted by $M$ as given by the following proposition.

Proposition 2: $d_{7}^{j}=M \times T_{B}^{j}$, with $\left.\left.M \in\right] 0.10\right]$.

Indeed, the multiplicity factor is a real number that is determined by the customer to determine the degree of impact of the price in the model. In certain situations, the customer don't care about price, he just give importance to other variables. In these situations, the customer specify small value to multiplicity factor. On the other hand, if the customer is limited to a certain budget and price is a must to him, for this case the customer specify a high value of the multiplicity factor. 
2) Size of company distance: The value of $d_{14}^{j}$ is in [0$10]$. For this reason, we propose the following measuring of corresponding distance $d_{14}^{j}$ for the variable "Size of company".

The distances of the variable $x_{14}$ will be presented in the Tables I, II, III and IV

TABLE I. SizE OF COMPANy DistANCE WHEN $X_{14}=M$

\begin{tabular}{|c|c|c|}
\hline$X_{14}$ & $Y_{14}(j)$ & $d_{14}^{j}$ \\
\hline & $M$ & 10 \\
$M$ & $L$ & 10 \\
& $S$ & 0 \\
& $N A$ & 0 \\
\hline
\end{tabular}

TABLE II. SiZE OF COMPANY DISTANCE WHEN $X_{14}=L$

\begin{tabular}{|c|c|c|}
\hline$X_{14}$ & $Y_{14}(j)$ & $d_{14}^{j}$ \\
\hline \multirow{4}{*}{$M$} & $M$ & 0 \\
& $L$ & 10 \\
& $S$ & 0 \\
& $N A$ & 0 \\
\hline
\end{tabular}

TABLE III. SIZE of COMPANy Distance WHEN $X_{14}=S$

\begin{tabular}{|c|c|c|}
\hline$X_{14}$ & $Y_{14}(j)$ & $d_{14}^{j}$ \\
\hline & $M$ & 10 \\
$M$ & $L$ & 10 \\
& $S$ & 10 \\
& $N A$ & 0 \\
\hline
\end{tabular}

TABLE IV. SIZE OF COMPANy Distance When $X_{14}=N A$

\begin{tabular}{|c|c|c|}
\hline$X_{14}$ & $Y_{14}(j)$ & $d_{14}^{j}$ \\
\hline & $M$ & 10 \\
$M$ & $L$ & 10 \\
& $S$ & 10 \\
& $N A$ & 10 \\
\hline
\end{tabular}

\section{B. Weights Calculation}

Due to the relative importance of the budget, variables related to price will be given more weights. The weights values will be calculated based on the econometric model by the $S P S S$ based on the previous offers calculations to specify the required t-student values for the specified variables.

\section{Scoring Formulation}

To help decision-makers, the offers are ranked for easier calculations. The previously defined weights and distances will be used to determine offers scoring values, these values will be in the range $[0-100]$. For each $Y$ element the distance $d_{i}^{j}$ between $Y_{i}(j)$ and the threshold value $T h_{i}$ must be calculated where $i \in\{4, \ldots, 15\}$. The customer (decision maker) specifies the threshold for each given offer $j$ Calculations of the distance are related to $\left\langle Y_{4}(j), \ldots, Y_{15}(j)\right\rangle$.

The following proposition explain the score for every given offer $j$ denoted by $S c_{j}$ and is as follows:

Proposition 3:

$$
S c_{j}=\frac{\sum_{4}^{15}\left(w_{i} \times d_{i}^{j}\right)}{\sum_{4}^{15} w_{i}} \times 10
$$

Proof: Each $d_{i}^{j}$ is in the interval of $[0-10]$. the multiplication of each $d_{i}^{j}$ by the corresponding weight $w_{i}$ for all $i \in\{4, \ldots, 15\}$ will be defined as the total weighted distance ( $T W D$ ). The resulted $T W D$ will be divided by $\sum_{4}^{15} w_{i}$ to obtain a value, multiplying this value by 10 will obtain $S c_{j}$.

Table VII, shows interval values descriptions:

TABLE V. INTERVAL SCORE VALUE DESCRIPTION

\begin{tabular}{|c|l|l|}
\hline Num & Rank interval & Description \\
\hline 1 & {$[0-20[$} & Rejected \\
2 & {$[20-40[$} & Not considered \\
3 & {$[40-50[$} & Below preferences \\
4 & {$[50-60[$} & Acceptable \\
5 & {$[60-70[$} & Good offer \\
6 & {$[70-80[$} & Very good \\
7 & {$[80-85[$} & Excellent \\
8 & {$[85-90[$} & Very excellent \\
9 & {$[90-95[$} & Exceptional \\
10 & {$[95-100]$} & Very exceptional \\
\hline
\end{tabular}

Example 4: Assume that the weights of each variable is given following:

$w_{4}=17.8 ; w_{5}=1.1 ; w_{6}=1.3 ; w_{7}=4.6 ; w_{8}=1.9 ; w_{9}=$ $1.6 ; w_{10}=2.1 ; w_{11}=1.9 ; w_{12}=3.1 ; w_{13}=1.2 ; w_{14}=$ $1.4 ; w_{15}=2.1$ For offer number 6 , assume we have the following calculated distances:

$d_{4}^{6}=9.8 ; d_{5}^{6}=3.3 ; d_{6}^{6}=10 ; d_{7}^{6}=0 ; d_{8}^{6}=7.8 ; d_{9}^{6}=$ $10 ; d_{10}^{6}=10 ; d_{11}^{6}=8 ; d_{12}^{6}=6.3 ; d_{13}^{6}=0 ; d_{14}^{6}=0 ; d_{15}^{6}=8$. $S c_{6}=\frac{\sum_{4}^{15}\left(w_{i} \times d_{i}^{6}\right)}{\sum 4^{15} w_{i}} \times 10=\frac{294.3}{40.1} \times 10=73.4$. Refer to Table $\mathrm{V}$, the $6^{t h}$ offer is a "Very good offer".

\section{D3S Algorithms}

that will be used by the this section presents 4 algorithms $D 3 S$ model. The first algorithm is devoted for the calculations of $S c_{j}$ score. while the second algorithm will be used to select the best offer. The evaluation of the best score determined by the manager will be calculated by the third algorithm. The last algorithm accurate all the previous algorithms to formulate D3S.

The next algorithm will be used to calculate all the distances $d_{i}^{j}$ and the vector weights $W$ in order to find the score $S c_{j}$ of the certain offer $j$

\begin{tabular}{l}
\hline Algorithm 1 Scoring $(Y(j))$ algorithm for calculation of the \\
$S c_{j}$ score \\
\hline 1: for $i=4$ to 15 do \\
2: $\quad$ Calculate $d_{i}^{j}$ \\
3: end for \\
4: $W=S P S S(X)$ \\
5: $S c_{j}=\frac{\sum_{i=15}^{i=4} w_{i} \times d_{i}^{j}}{\sum_{i=15}^{i=4} w_{i}}$ \\
6: Stop. Return $S c_{j}$. \\
\hline
\end{tabular}




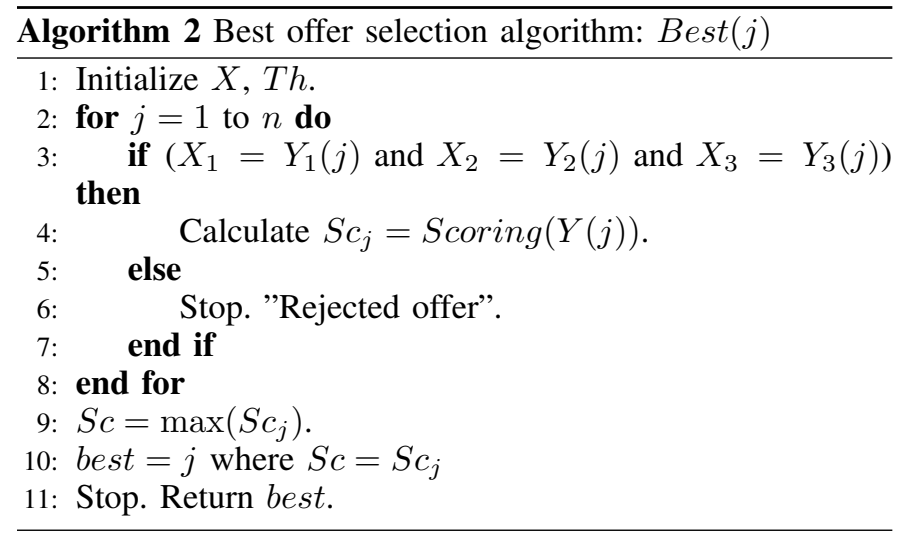

After getting the best scores for the given $n$ offers. The next step is to evaluate the description of the offer referring to the Table VII. Suppose that the latter table is saved in a pointer table named Desc. We denote by $\operatorname{Desc}\left(S c_{j}\right)$ (Description of the obtained score) the function that devoted to return the description of the score given as an input of the function refereeing to the table pointer $\operatorname{Desc}_{s} c$.

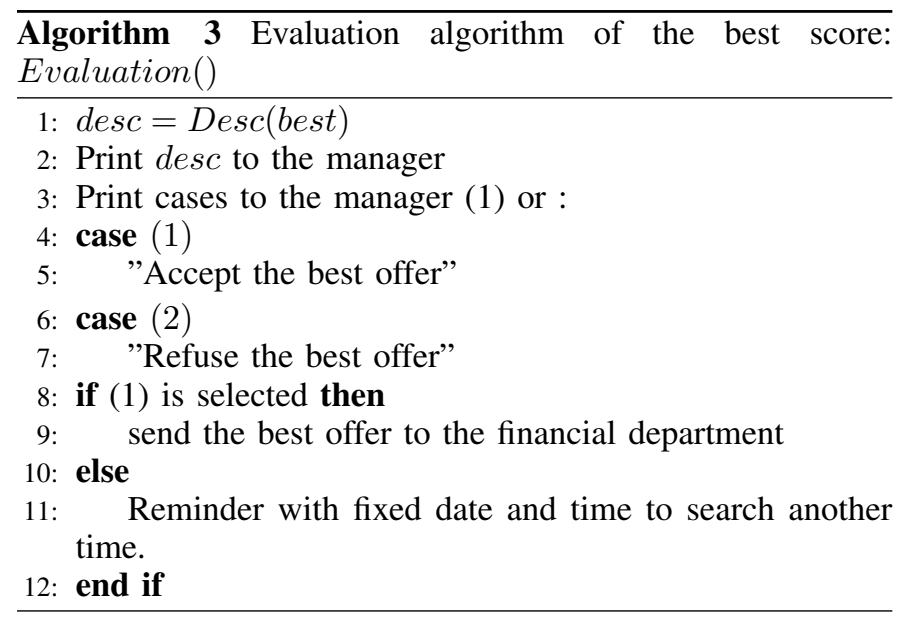

Now we use the previous algorithms to generate the $D 3 S$ final algorithm as follows:

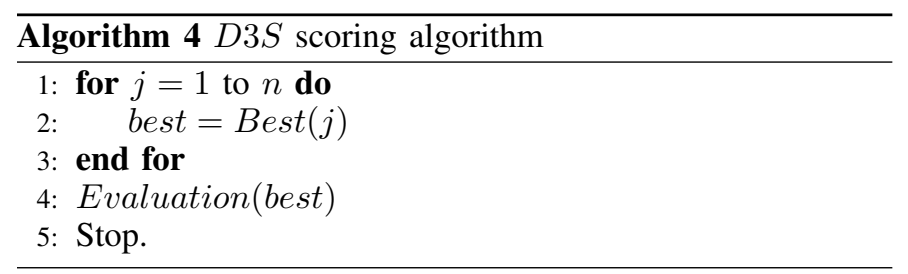

\section{EXPERIMENTAL RESULtS}

For more explanations the given algorithms were implemented by Microsoft Visual C++ (Version 2012). The implemented algorithms were executed by 8GB RAM i5 PC. Decisive variables in these experiments are as follows: $X_{1}=P c$; $X_{2}=H P ; X_{3}=x a 03 n f$. We received a total of 55 offers. 28 of the received offers were rejected after applying decisive variables leaving 27 offers for evaluation.
The customer specifies the maximum budget by $4,000 \$$. Due to this limitation 17 out of the remaining 27 offers will be dropped leaving only 10 offers to be considered. the values regarding vector $X$ and $Y(j)$ elements with $j \in\{1, \ldots, 10\}$ are shown in Table VI below. The 10 offers weight and distances are shown in Table VII. Finally, Table VIII represents the 10 pre-selected offers derived scores.

TABLE VI. OFFER AND PREFERENCES

\begin{tabular}{|c|c|c|c|c|c|c|c|}
\hline & \multirow{2}{*}{\multicolumn{2}{|c|}{ Preferences }} & \multicolumn{5}{|c|}{ Offer } \\
\hline & & & 1 & 2 & $\mathbf{3}$ & 4 & 5 \\
\hline \multirow{15}{*}{$T H$} & 1 & PC & $\overline{P C}$ & PC & $\overline{\mathrm{PC}}$ & PC & PC \\
\hline & 2 & HP & HP & HP & HP & HP & HP \\
\hline & 3 & $\mathrm{xa03nf}$ & xa03nf & $\mathrm{xa} 03 \mathrm{nf}$ & $\mathrm{xa03nf}$ & $\mathrm{xa03nf}$ & xa03nf \\
\hline & 4 & 50 & 54 & 43 & 52 & 30 & 50 \\
\hline & 5 & 3 & 2 & 1 & 2 & 3 & 4 \\
\hline & 6 & 2 & 1 & 2 & 3 & 1 & 2 \\
\hline & 7 & 3354 & 3100 & 3210 & 3258 & 3548 & 3344 \\
\hline & 8 & 9 & 8 & 7 & 9 & 10 & 11 \\
\hline & 9 & 3 & 2 & 1 & 3 & 3 & 2 \\
\hline & 10 & 1 & 1 & 1 & 1 & 1 & 2 \\
\hline & 11 & 5 & 5 & 4 & 3 & 5 & 3 \\
\hline & 12 & 8 & 8 & 7 & 8 & 4 & 4 \\
\hline & 13 & yes & yes & yes & No & NA & NA \\
\hline & 14 & $\mathrm{M}$ & $\mathrm{M}$ & $\mathrm{L}$ & $\mathrm{S}$ & $\mathrm{S}$ & NA \\
\hline & 15 & 5 & 5 & 8 & 2 & 3 & 2 \\
\hline
\end{tabular}

\begin{tabular}{|c|c|c|ccccc|}
\cline { 2 - 8 } \multicolumn{1}{c|}{} & \multicolumn{2}{|c|}{ Preferences } & \multicolumn{6}{c|}{ Offer } \\
\cline { 2 - 7 } & $\mathbf{1}$ & PC & PC & PC & PC & PC & PC \\
\hline \multirow{5}{*}{ TH } & HP & HP & HP & HP & HP & HP \\
& $\mathbf{3}$ & xa03nf & xa03nf & xa03nf & xa03nf & xa03nf & xa03nf \\
\cline { 2 - 7 } & $\mathbf{4}$ & 50 & 49 & 52 & 50 & 53 & 22 \\
& $\mathbf{5}$ & 3 & 1 & 2 & 3 & 1 & 4 \\
& $\mathbf{6}$ & 2 & 3 & 4 & 3 & 2 & 1 \\
& $\mathbf{7}$ & 3354 & 3351 & 3245 & 3158 & 3111 & 3987 \\
& $\mathbf{8}$ & 9 & 7 & 9 & 8 & 12 & 4 \\
& $\mathbf{9}$ & 3 & 3 & 2 & 1 & 2 & 2 \\
& $\mathbf{1 0}$ & 1 & 2 & 1 & 2 & 1 & 1 \\
& $\mathbf{1 1}$ & 5 & 4 & 5 & 3 & 4 & 4 \\
& $\mathbf{1 2}$ & 8 & 5 & 6 & 7 & 7 & 7 \\
& $\mathbf{1 3}$ & yes & No & yes & NA & No & NA \\
& $\mathbf{1 4}$ & M & S & L & M & NA & L \\
& $\mathbf{1 5}$ & 5 & 4 & 20 & 10 & 0 & 1 \\
\hline
\end{tabular}

TABLE VII. WEIGHTS AND DISTANCES

\begin{tabular}{|c|c|c|c|c|c|c|}
\hline & \multirow{2}{*}{$w_{i}$} & \multicolumn{5}{|c|}{$d_{i}^{j}$} \\
\hline & & 1 & 2 & 3 & 4 & 5 \\
\hline 4 & 17.8 & 10.0 & 8.6 & 10.0 & 6.0 & 10.0 \\
\hline 5 & 1.1 & 6.7 & 3.3 & 6.7 & 10.0 & 10.0 \\
\hline 6 & 1.3 & 5.0 & 10.0 & 10.0 & 5.0 & 10.0 \\
\hline 7 & 4.6 & 0.8 & 0.4 & 0.3 & -0.6 & 0.0 \\
\hline 8 & 1.9 & 8.9 & 7.8 & 10.0 & 10.0 & 10.0 \\
\hline 9 & 1.6 & 6.7 & 3.3 & 10.0 & 10.0 & 6.7 \\
\hline 10 & 2.1 & 10.0 & 10.0 & 10.0 & 10.0 & 10.0 \\
\hline 11 & 1.9 & 10.0 & 8.0 & 6.0 & 10.0 & 6.0 \\
\hline 12 & 3.1 & 10.0 & 8.8 & 10.0 & 5.0 & 5.0 \\
\hline 13 & 1.2 & 10.0 & 10.0 & 0.0 & 0.0 & 0.0 \\
\hline 14 & 1.4 & 10.0 & 10.0 & 0.0 & 0.0 & 0.0 \\
\hline 15 & 2.1 & 10.0 & 10.0 & 4.0 & 6.0 & 4.0 \\
\hline
\end{tabular}

\begin{tabular}{|c|c|c|c|c|c|c|}
\hline & \multirow[b]{2}{*}{$w_{i}$} & \multicolumn{5}{|c|}{$d_{i}^{j}$} \\
\hline & & 6 & 7 & $\mathbf{8}^{2}$ & 9 & 10 \\
\hline 4 & 17.8 & 9.8 & 10.0 & 10.0 & 10.0 & 4.4 \\
\hline 5 & 1.1 & 3.3 & 6.7 & 10.0 & 3.3 & 10.0 \\
\hline 6 & 1.3 & 10.0 & 10.0 & 10.0 & 10.0 & 5.0 \\
\hline 7 & 4.6 & 0.0 & 0.3 & 0.6 & 0.7 & -1.9 \\
\hline 8 & 1.9 & 7.8 & 10.0 & 8.9 & 13.3 & 4.4 \\
\hline 9 & 1.6 & 10.0 & 6.7 & 3.3 & 6.7 & 6.7 \\
\hline 10 & 2.1 & 10.0 & 10.0 & 10.0 & 10.0 & 10.0 \\
\hline 11 & 1.9 & 8.0 & 10.0 & 6.0 & 8.0 & 8.0 \\
\hline 12 & 3.1 & 6.3 & 7.5 & 8.8 & 8.8 & 8.8 \\
\hline 13 & 1.2 & 0.0 & 10.0 & 0.0 & 0.0 & 0.0 \\
\hline 14 & 1.4 & 0.0 & 10.0 & 10.0 & 0.0 & 10.0 \\
\hline 15 & 2.1 & 8.0 & 10.0 & 10.0 & 0.0 & 2.0 \\
\hline
\end{tabular}


TABLE VIII. SCORING OFFERS

\begin{tabular}{|c|c|c|}
\cline { 2 - 3 } \multicolumn{1}{c|}{} & Index & $S c_{i}$ \\
\hline \multirow{6}{*}{ Offer } & 1 & 85.0 \\
& 2 & 75.4 \\
& 3 & 76.4 \\
& 4 & 56.0 \\
& 5 & 71.8 \\
& 6 & 73.4 \\
& 7 & 84.7 \\
& 8 & 80.2 \\
& 9 & 74.1 \\
& 10 & 46.8 \\
\hline
\end{tabular}
V:

The description of each offer will be presented by Table

- Offer 1: Very excellent

- Offer 2: Very good

- Offer 3: Very good

- Offer 4: Acceptable

- Offer 5: Very good

- Offer 6: Very good

- Offer 7: Exceptional

- Offer 8: Excellent

- Offer 9: Very good

- Offer 10: Below preferences

Based on the given valued the chosen offer (the best offer) is offer 7 .

\section{CONCLUSION}

In order to cope with the technological, economical and political challenges, in the case of supply chain management and e-procurement; decision makers should consider all these limitations when evaluating or selecting a given offer. To highlight this challenge, this paper presented an intelligent $D 3 S$ model, which consists of 4 developed algorithms and preliminary $D 3 S$ model. The developed $D 3 S$ model is based on multi-criteria evaluation and selection intended to be used as a decision support system, that can be utilized by the decision makers in their evaluation during the supply chain management or the e-procurement processes. Experimental results showed that the deployment of the $D 3 S$ derived the best offer among a set of presented offers while considering a given determinants specified by the customer.

\section{ACKNOWLEDGMENT}

The authors would like to thank the Deanship of Scientific Research at Majmaah University for supporting this work under Project Number No. 1440-88

\section{REFERENCES}

[1] A. Aalaei and H. Davoudpour, "Revised multi-choice goal programming for incorporated dynamic virtual cellular manufacturing into supply chain management: A case study," Engineering Applications of Artificial Intelligence, vol. 47, pp. 3-15, 2016.

[2] F. Çebi and İ. Otay, "A two-stage fuzzy approach for supplier evaluation and order allocation problem with quantity discounts and lead time," Information Sciences, vol. 339, pp. 143-157, 2016.
[3] A. Gunasekaran, Z. Irani, K.-L. Choy, L. Filippi, and T. Papadopoulos, "Performance measures and metrics in outsourcing decisions: A review for research and applications," International Journal of Production Economics, vol. 161, pp. 153-166, 2015.

[4] K. Govindan, S. Rajendran, J. Sarkis, and P. Murugesan, "Multi criteria decision making approaches for green supplier evaluation and selection: a literature review," Journal of Cleaner Production, vol. 98, pp. 66-83, 2015.

[5] F. R. Lima-Junior and L. C. R. Carpinetti, "A multicriteria approach based on fuzzy qfd for choosing criteria for supplier selection," Computers \& Industrial Engineering, vol. 101, pp. 269-285, 2016.

[6] S. Bali and S. S. Amin, "An analytical framework for supplier evaluation and selection: a multi-criteria decision making approach," International Journal of Advanced Operations Management, vol. 9, no. 1, pp. 57-72, 2017.

[7] M. Jemmali, M. Alharbi, and L. K. B. Melhim, "Intelligent decisionmaking algorithm for supplier evaluation based on multi-criteria preferences," in 2018 1st International Conference on Computer Applications \& Information Security (ICCAIS). IEEE, 2018, pp. 1-5.

[8] A. K. Kar, "Revisiting the supplier selection problem: An integrated approach for group decision support," Expert systems with applications, vol. 41, no. 6, pp. 2762-2771, 2014.

[9] A. Zouggari and L. Benyoucef, "Simulation based fuzzy topsis approach for group multi-criteria supplier selection problem," Engineering Applications of Artificial Intelligence, vol. 25, no. 3, pp. 507-519, 2012.

[10] F. Dweiri, S. Kumar, S. A. Khan, and V. Jain, "Designing an integrated ahp based decision support system for supplier selection in automotive industry," Expert Systems with Applications, vol. 62, pp. 273-283, 2016.

[11] S. Brownlow and S. Watson, "Structuring multi-attribute value hierarchies," Journal of the Operational Research Society, vol. 38, no. 4, pp. 309-317, 1987.

[12] A. Naseem, S. T. H. Shah, S. A. Khan, and A. W. Malik, "Decision support system for optimum decision making process in threat evaluation and weapon assignment: Current status, challenges and future directions," Annual reviews in control, vol. 43, pp. 169-187, 2017.

[13] A. Mardani, A. Jusoh, and E. K. Zavadskas, "Fuzzy multiple criteria decision-making techniques and applications-two decades review from 1994 to 2014," Expert systems with Applications, vol. 42, no. 8, pp. 4126-4148, 2015.

[14] R. M. Rodríguez, Á. Labella, and L. Martínez, "An overview on fuzzy modelling of complex linguistic preferences in decision making," International Journal of Computational Intelligence Systems, vol. 9, no. sup1, pp. 81-94, 2016.

[15] L. Ferreira, D. Borenstein, and E. Santi, "Hybrid fuzzy madm ranking procedure for better alternative discrimination," Engineering Applications of Artificial Intelligence, vol. 50, pp. 71-82, 2016.

[16] B. Vahdani, S. M. Mousavi, H. Hashemi, M. Mousakhani, and R. Tavakkoli-Moghaddam, "A new compromise solution method for fuzzy group decision-making problems with an application to the contractor selection," Engineering Applications of Artificial Intelligence, vol. 26, no. 2, pp. 779-788, 2013.

[17] J. Carvalho, M. L. R. Varela, G. D. Putnik, J. E. Hernández, and R. A. Ribeiro, "A web-based decision support system for supply chain operations management towards an integrated framework," in Decision Support Systems III-Impact of Decision Support Systems for Global Environments. Springer, 2013, pp. 104-117.

[18] W. Ho, X. Xu, and P. K. Dey, "Multi-criteria decision making approaches for supplier evaluation and selection: A literature review," European Journal of operational research, vol. 202, no. 1, pp. 16-24, 2010.

[19] S. Shukla, P. Mishra, R. Jain, and H. Yadav, "An integrated decision making approach for erp system selection using swara and promethee method," International Journal of Intelligent Enterprise, vol. 3, no. 2, pp. 120-147, 2016.

[20] P. Toktaş-Palut, E. Baylav, S. Teoman, and M. Altunbey, "The impact of barriers and benefits of e-procurement on its adoption decision: An empirical analysis," International Journal of Production Economics, vol. 158, pp. 77-90, 2014. 